\title{
Experimental comparison of terahertz and infrared data signal attenuation in dust clouds
}

\author{
Ke Su, ${ }^{1, *}$ Lothar Moeller, ${ }^{2}$ Robert B. Barat,${ }^{3}$ and John F. Federici ${ }^{1}$ \\ ${ }^{1}$ Department of Physics, New Jersey Institute of Technology, 322 King Blvd., Newark, New Jersey 07102, USA \\ ${ }^{2}$ Bell Laboratories/Alcatel-Lucent, 791 Holmdel-Keyport Road, Holmdel, New Jersey 07733, USA \\ ${ }^{3}$ Otto York Department of Chemical Engineering, New Jersey Institute of Technology, 322 King Blvd., \\ Newark, New Jersey 07102, USA \\ *Corresponding author: ks265@njit.edu
}

Received July 6, 2012; revised September 26, 2012; accepted September 27, 2012; posted September 27, 2012 (Doc. ID 172090); published October 18, 2012

\begin{abstract}
In order to study and compare propagation features of terahertz (THz) links with infrared (IR) links under different weather conditions such as turbulence, fog, and dust particles, $\mathrm{THz}$ and $\mathrm{IR}$ free space communication links at $625 \mathrm{GHz}$ carrier frequency and $1.5 \mu \mathrm{m}$ wavelength, respectively, with a maximum data rate of $2.5 \mathrm{~Gb} / \mathrm{s}$ have been developed. After propagating through the same channel perturbation caused by dust, attenuation of the carrier frequencies by dust as well as scintillation effects on both channels are analyzed by measuring the power attenuation and bit error rates. Attenuation by the presence of dust degrades the IR channel but exhibits almost no measurable impact on the THz signal. Numerical simulations of $\mathrm{THz}$ attenuation with different dust concentrations are presented and agree with the measured results. (c) 2012 Optical Society of America
\end{abstract}

OCIS codes: $\quad 060.2605,040.2235,140.3070,290.5930,010.1300$.

\section{INTRODUCTION}

Recently, terahertz (THz) communication has attracted more attention because of its potential to support higher data rates $[\underline{1}, 2]$. According to Edholm's law of bandwidth [3] , data rates of $5-10 \mathrm{~Gb} / \mathrm{s}$ will be needed 10 years from now. The increasing demand of higher data rates for wireless communication systems will likely lead soon to operational systems in the lower $\mathrm{THz}$ frequency range.

Both indoor and outdoor scenarios of THz communications have been considered. Research on indoor THz links has focused on various important channel features, and several experiments have been conducted [4-]]. Koch and his coworkers mainly focus on the ray-tracing simulations of $\mathrm{THz}$ indoor communication [8]. However, our focus is on performance degradation of outdoor $\mathrm{THz}$ communication under certain weather conditions such as humidity, rain, fog, dust, and scintillation effects. Towards higher carrier frequencies, $\mathrm{THz}$ can possibly enable reliable signaling at certain atmospheric conditions where free space infrared (IR) communication would fail. Some experiments have been done to investigate the impact of weather conditions on the THz transmission link. For example, Yang et al. [9] recently characterize attenuation of the water vapor form 0.2 to $2 \mathrm{THz}$ using $\mathrm{THz}$ time-domain spectroscopy and show higher attenuation than previously measured or predicted in these $\mathrm{THz}$ transmission windows. At a frequency region of above $100 \mathrm{GHz}$, the effect of rain has been characterized at $103 \mathrm{GHz}$ [10], $120 \mathrm{GHz}$ [11], and $355.2 \mathrm{GHz}$ [12]. Yamaguchi et al. [13] show the measurement of scintillations due to wind effects on a propagating $125 \mathrm{GHz}$ data signal. Recently, we performed a direct experimental performance comparison between THz and IR communication links through fog, meaning that both signals experience the same fog conditions [14]. Mann [15] predicts that smoke has little or no effect up to $1 \mathrm{THz}$ due to the relatively small size of atmospheric particulates compared to $\mathrm{THz}$ wavelengths. Federici and Moeller [1] theoretically estimate attenuation due to smoke particles for both $\mathrm{THz}$ and IR range and conclude that IR wavelengths are strongly attenuated while $\mathrm{THz}$ radiation can propagate well through small airborne particulates such as dust. However, to the best of our knowledge, no experimental study of the attenuation from $\mathrm{THz}$ data signal in dust has been published. We focus here on channel impairments caused by dust, which are mainly due to particle scattering and refractive index fluctuation (scintillation effects).

As discussed in [1] , local temperature, pressure, or humidity gradients, which are generated by thermals and turbulences near ground level, cause small real refractive index fluctuations across the wave front of the beam. The fluctuations of the real refractive index through the path of a beam can cause random deflection and interference between different portions of the wavefront and destroy the flat phase front of an IR light beam; therefore, the beam cross section on the receiver side appears as a speckle pattern (Fig. 1) with huge local and temporal intensity variations. Scintillation effects are a major limitation to the maximum transmission distance limitation of IR communication links. According to the analysis in [1], since optical path length variations in the THz and IR ranges are comparable, the relative magnitudes of the phase variations are predominately determined by the electromagnetic wavelength. The wavelength of $\mathrm{THz}$ at $\sim 625 \mathrm{GHz}$ is approximately 320 times longer than the wavelength of $1.5 \mu \mathrm{m}$ light. Since scintillation effects are driven by phase variation, consequently, scintillation and speckle effects in the $\mathrm{THz}$ beam are expected to be significantly smaller than in the IR.

A THz and IR communications lab setup with a maximum data rate of $2.5 \mathrm{~Gb} / \mathrm{s}$ at $625 \mathrm{GHz}$ carrier frequency and $1.5 \mu \mathrm{m}$ wavelength has been developed [14]. The performance 


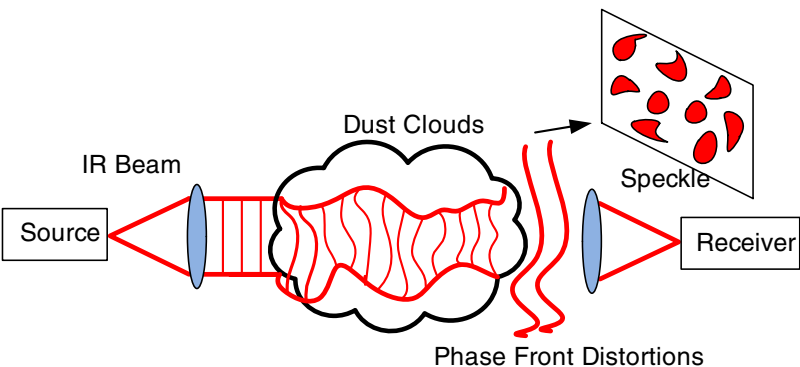

Fig. 1. (Color online) Air turbulence causes refractive index fluctuations resulting in speckle (intensity variations at receiver).

degradation in both channels due to dust attenuation can be simultaneously recorded and analyzed. We investigate attenuation and scintillation effects caused by dust through bit error rate (BER) performance and received power levels. Our investigations show for the first time how differently $\mathrm{THz}$ and IR communication signals are degraded when passing through the same dust conditions. Simulations of THz attenuation with different dust concentrations are presented and show good agreement with the experimental measurements.

\section{EXPERIMENTAL SETUP}

Figure 2(a) shows a block diagram and Fig. 2(b) shows a photo of our experimental setup. Detailed features of the
THz link $[\underline{7}, \underline{14}]$ and IR link [14] were presented earlier. In the present work, we focus on the details of the links which are important for the communication channels through dust.

As shown in Fig. 2(a), the output of the horn antenna from the $\mathrm{THz}$ transmitter is collimated by a $\mathrm{THz}$ lens with short focal length $(\sim 32 \mathrm{~mm})$ to a beam with $\sim 20 \mathrm{~mm}$ diameter, transmitted over a few meters distance and finally coupled into a $\mathrm{THz}$ receiver horn similar to the transmitter antenna. A $2.5 \mathrm{~Gb} / \mathrm{s}$ non-return-to-zero (NRZ) format signal is generated by a pulse pattern generator. A duobinary modulation technique $[\underline{16}, \underline{17}]$ is utilized in the system for driving the $\mathrm{THz}$ source, which enables signaling at high data rate, with relatively compact spectrum and higher output power. An iris with $8.5 \mathrm{~mm}$ aperture, inserted concentrically into the beam, limits its total power to an amount that results in a BER of about $10^{-6}$ for an unloaded dust chamber. We chose to adjust our setup at a BER of $10^{-6}$ because statistical fluctuations of the BER at levels of $10^{-6}$ and higher do not significantly impact conclusions we draw from our measurement results [14]. Also, BER of $10^{-6}$ is a typical threshold for modern forward error correction technology used in lightwave communication systems [18].

The IR transmitter is driven by the same $2.5 \mathrm{~Gb} / \mathrm{s}$ NRZ data pattern as our THz source. A 90:10 single mode fiber coupler launches a small fraction of the signal into photo detector PD_1, which serves as monitor for the power entering the dust

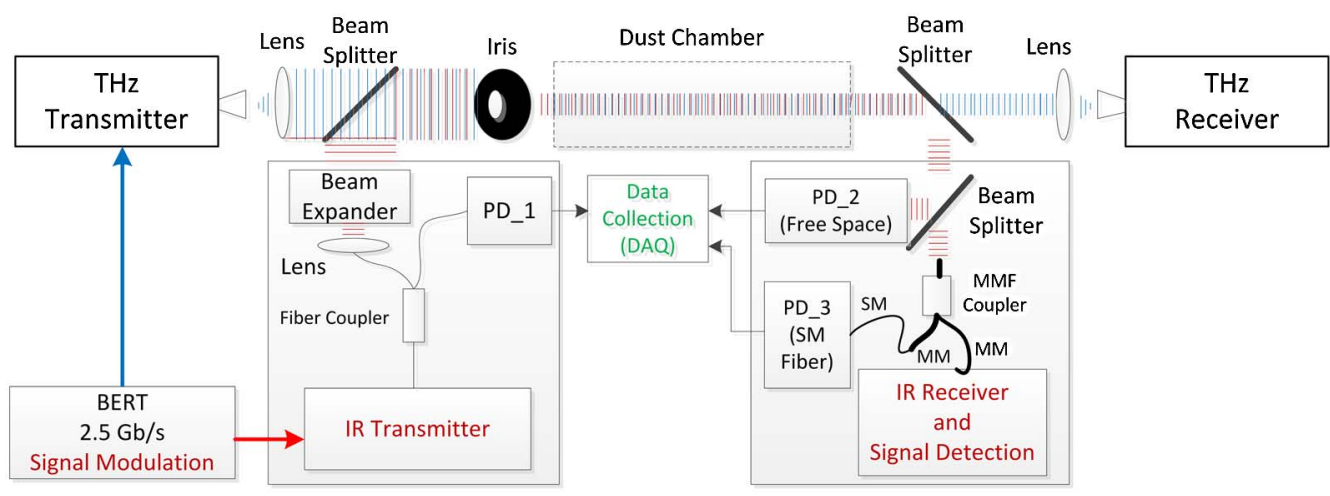

(a)

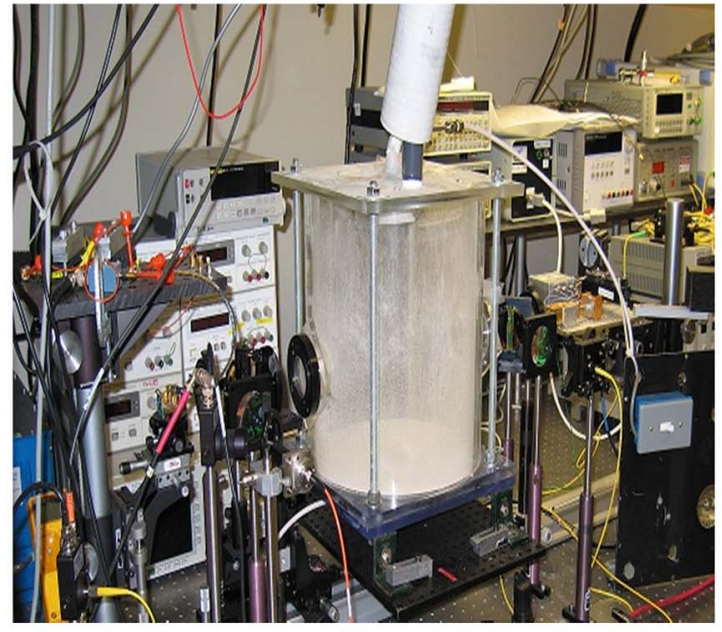

(b)

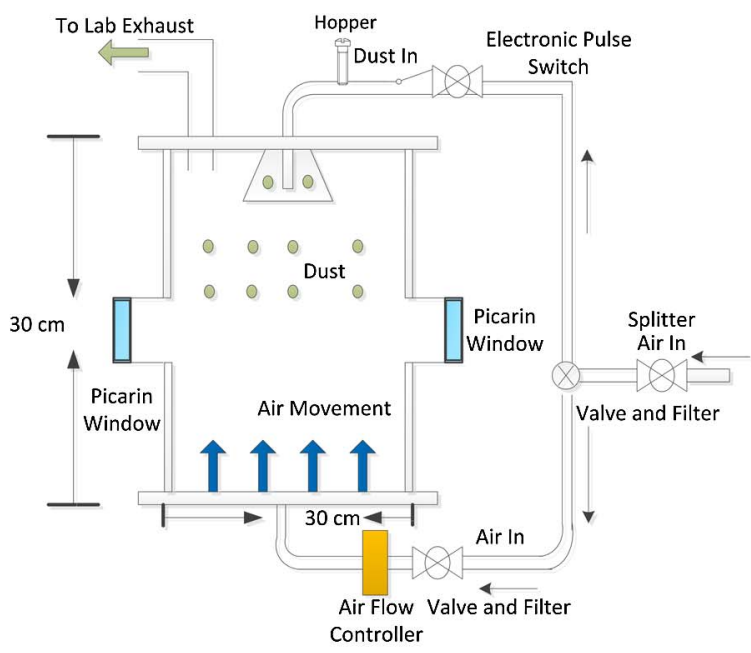

(c)

Fig. 2. (Color online) (a) Schematic diagram of the THz and IR wireless communication link through a dust chamber, (b) photo of setup, and (c) schematic diagram of the dust chamber design. 
chamber. We expand the IR beam to about $20 \mathrm{~mm}$ diameter by a fiber collimator, which is comparable to the $\mathrm{THz}$ beam size before the iris. The collimated IR beam is superimposed with the $\mathrm{THz}$ beam using a thin $(2 \mu \mathrm{m})$ nitrocellulose membrane beam splitter (Thorlabs) with $55 \%$ reflection ratio at $45^{\circ}$ incident angle, and transmitted through the dust chamber. After emerging from the chamber, the beam is deflected with a similar beam splitter to spatially separate THz and IR signals. A second beam splitter taps off a fraction of the IR power leaving the chamber and launches it towards a large area photo diode (effective area $19.6 \mathrm{~mm}^{2}$ ) of detector PD_2 which, in combination with PD_1, is used to determine the power loss caused by the chamber load. The remaining beam power enters via a fiber collimator a $1 \times 2$ multimode $(\mathrm{MM})$ fiber coupler with 50:50 splitting ratio. Its output power is launched to a low bandwidth photo detector (PD_3) with a $1 \mathrm{~m}$ long standard single mode fiber (SSMF) and to a dccoupled IR lightwave converter (Agilent 81495A) with $9 \mathrm{GHz}$ bandwidth. The lightwave converter which is accessible via general purpose interface bus (GPIB) is used both for data detection and measuring the optical power of the incoming signal.

The output of PD_1, PD_2, and PD_3 are recorded via a DAQ board with 16 bit resolution and maximum $10 \mathrm{kHz}$ sampling rate. This sampling rate is sufficiently high to track even the fastest fluctuations of the signals. BERs, Radio-Frequency (RF) power of the THz signal, and optical power of the IR signal are recorded via GPIB. We set the LabView time controller at a clock rate of $500 \mathrm{~ms}$ to synchronize all recordings.

Our dust chamber is shaped like a cylinder with $30 \mathrm{~cm}$ diameter, $30 \mathrm{~cm}$ height, with top and bottom plates. Figure 2(c) shows the schematic diagram of the dust chamber design. A known total mass of dust particles is placed inside the hopper, which sits above the dust holder. An electronic pulse switch controls an air valve and the released air removes the dust from the dust holder and ejects it at a high speed into the chamber from the top plate of the dust chamber. The hopper can be easily refilled and it produces a fairly constant and reproducible feed of dust. Air flows into the chamber with constant volume speed through the holes from the bottom plate. The holes, all with the same $0.18 \mathrm{~cm}$ diameter, are placed on 5 concentric set of circles with diameters 2.22, 4.76, 7.30, 9.84, and $12.38 \mathrm{~cm}$. The constant air volume flow rate is regulated by an air flow controller to achieve specific flow rates. Two Picarin windows which are highly transparent to both the $\mathrm{THz}$ and IR beam are mounted at the input and output of the chamber. After experiments are completed, the dusty air can be extracted by the exhaust pump which is installed on the top plate.

Bentonite powder is a mixture of clay formed from volcanic ash decomposition and largely composed of montmorillonite and beidellite. We use bentonite as dust particles for loading the chamber. The average particle radius is $4.3 \mu \mathrm{m}$.

\section{EXPERIMENTAL RESULTS}

\section{A. Signal Attenuation by Dust}

After we launched constant IR power into the dust chamber, a typical evolution of the attenuations in both $\mathrm{THz}$ and IR channels is shown in Fig. 3(a). When $0.08 \mathrm{~g}$ of dust is launched in the propagation path of the beams, the maximum attenuation (around $4.5 \mathrm{~dB}$ ) of the IR light is clearly evident while the
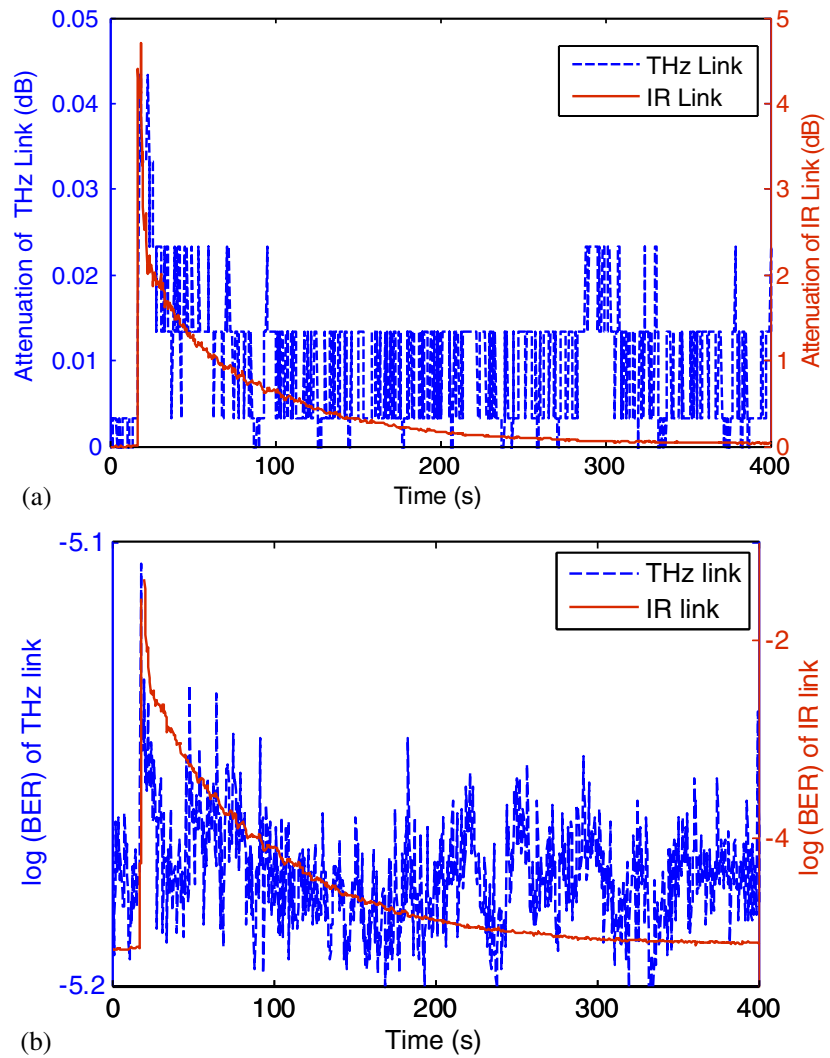

Fig. 3. (Color online) (a) Attenuation of THz link and IR link as the function of time, (b) $\log (\mathrm{BER})$ of THz link and IR link as the function of time.

impact on $\mathrm{THz}$ could be neglected (only $0.045 \mathrm{~dB}$ ). As will be shown by simulation (Section 4 ), the particle concentration is estimated to be roughly $4 \times 10^{9} / \mathrm{m}^{3}$. The attenuation decreases with time since dust particles start falling down to the bottom of the chamber. After 400 seconds, the IR transmitted power recovers and approaches its original performance. Our $\mathrm{RF}$ power meter is only accurate to within $0.01 \mathrm{~dB}$, which explains the rough quantization of the recorded $\mathrm{THz}$ signal in Fig. 3(a). For the IR channel, a relationship between the attenuation and the recorded BERs [Fig. 3(b)] is clearly visible. The recorded BERs for the THz link in Fig. 3(b) verify again that dust particles at our concentration levels $\left(4 \times 10^{9} / \mathrm{m}^{3}\right)$ have little impact on the THz signal.

\section{B. Comparison of Different Concentrations of Dust}

Three different amounts of dust $(0.05,0.08$, and $0.13 \mathrm{~g}$ ) are loaded in the dust chamber. Assuming injected dust particles are dispersed equally over the volume of the chamber, the dust concentrations can be estimated using

$$
N_{s}=\frac{N}{V_{\text {chamber }}}=\left(\frac{m_{p}}{V_{p} \rho_{p}}\right) / V_{\text {chamber }}=\left(\frac{m_{p}}{\frac{4}{3} \pi r^{3} \rho_{p}}\right) / V_{\text {chamber }}
$$

where $N$ is number of particles, $V_{\text {chamber }}$ is the volume of the chamber, $m_{p}$ is total mass of the dust that is loaded into the chamber, $\rho_{p}$ is the mass density of the dust $\left(2.7 \mathrm{~g} / \mathrm{cm}^{2}\right), V_{p}$ is the volume of a dust particle, and $r$ is the radius of the particle. The corresponding concentrations for different amounts of loaded dust $\left(0.05,0.08\right.$, and $0.13 \mathrm{~g}$ ) are $2.5 \times 10^{9} / \mathrm{m}^{3}$, 

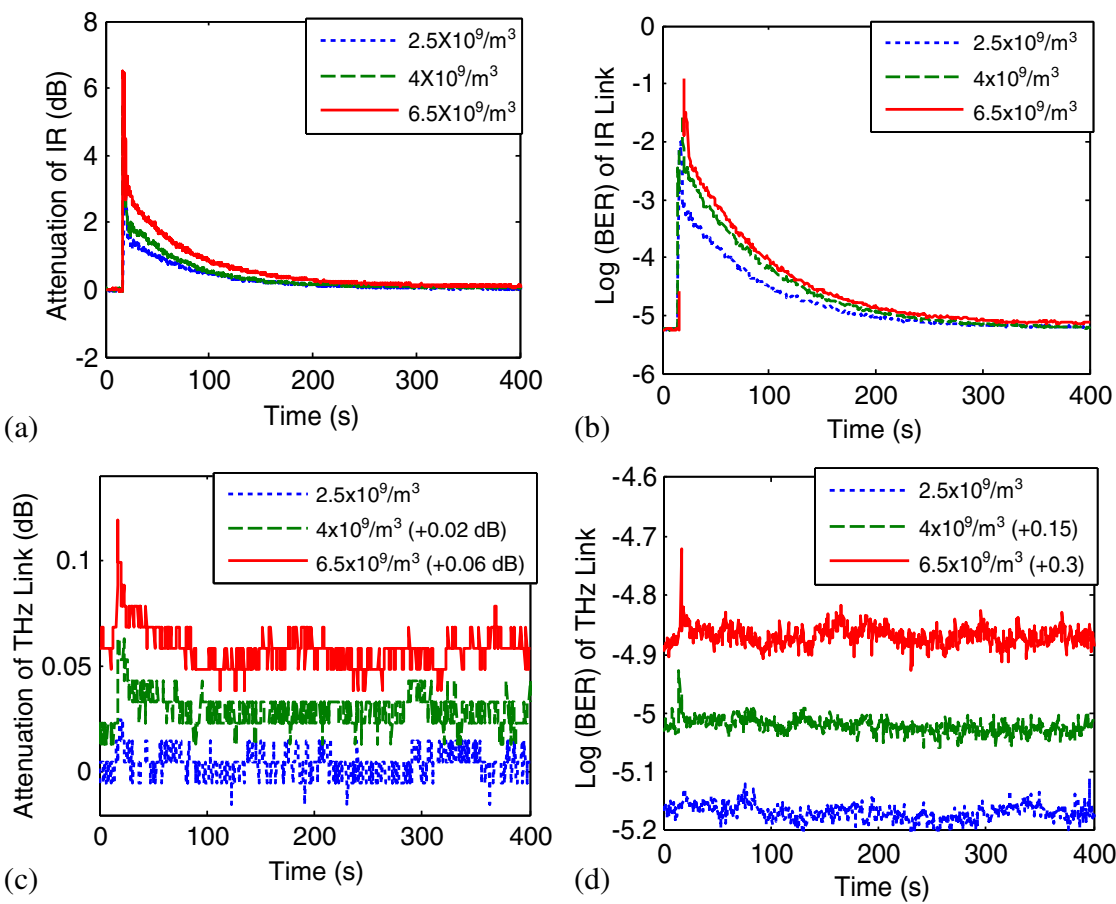

Fig. 4. (Color online) (a) Attenuation of IR link for different dust concentrations, (b) Log(BER) of IR link for different dust concentrations, (c) Attenuation of THz link for different dust concentrations, (d) Log(BER) of THz link for different dust concentrations (offset with the values mentioned in the legend).

$4 \times 10^{9} / \mathrm{m}^{3}$, and $6.5 \times 10^{9} / \mathrm{m}^{3}$. Attenuation of the IR signal and the corresponding BER as a function of time are measured for various dust amounts [Figs. 4(a) and 4(b)]. As expected, the attenuations vary significantly with changing particle concentration. The attenuation of the THz link and corresponding BER as a function of time are recorded as well [Figs. 4(c)-4(d)]. In order to better visualize the dynamic response, an offset has been added for the measurements corresponding to dust particle concentration of $4 \times 10^{9} / \mathrm{m}^{3}$ and $6.5 \times 10^{9} / \mathrm{m}^{3}$. The $\mathrm{THz}$ signal exhibits only a minor decrease in power.

\section{Scintillation Impact on IR Link}

In addition to the attenuation effects discussed above, scintillation effects could also cause an IR power variation on the receiver side. Because of the constantly changing dust pattern, scintillation appears to be more significant when the receiver has a small aperture. If scintillation effects were to occur, we would expect more pronounced variations in the power coupled into SSMF with small aperture (PD_3) compared to the output of the free space large area detector (PD_2) and MM fiber detector.

The variances of the detector signal can be further associated with three different sources: the electronic noise as well as scintillation effects caused by dust particles, and air flow inside the chamber. Air flow generates fluctuations of the optical detector output due to refraction index changes even when no dust particles are launched. This noise contribution we assess by the variance $\sigma_{\text {air }}^{2}$. Fluctuations caused by scintillation effects stemming from the dust particles we account for by $\sigma_{\text {dust-scintillation. Since all noise contributions }}^{2}$ are independent random processes, the total variance of the voltage fluctuation $\sigma_{\text {total }}^{2}$ at the detector output can be obtained by adding individual variances. However, except for the electronic noise, the individual contributions to variance depend on the optical power and detector features like its responsivity. To allow a fair comparison of the output fluctuations from the three different detectors, we normalize their variances with the low pass filtered and squared voltage value $V^{2}(t)$ of their outputs. Thus, the normalized variance of total detector output noise is given by

$$
\begin{aligned}
\sigma_{\text {total }}^{2} / V^{2}(t)= & \sigma_{\text {electronics }}^{2} / V^{2}(t)+\sigma_{\text {air }}^{2} / V_{\text {initial }}^{2} \\
& +\sigma_{\text {dust-scintillation }}^{2} / V_{\text {initial }}^{2},
\end{aligned}
$$

where $V_{\text {initial }}$ stands for the detector output voltage at the beginning of the experiment, when no dust particles were launched but air is flowing through the chamber. The low pass filtering of the detector output is chosen such that relatively fast fluctuations stemming from electronic noise are suppressed whereas slow processes like the decay of the dust particle concentration and scintillations can be well captured [Figs. 5(a) and 5(b)]. The filtering is performed by convoluting the detector output with a rectangular impulse response which leads to an averaging of 100 adjacent sampling points from DAQ board output.

In our experience, at high sampling rates $(\sim 10 \mathrm{kHz}), \mathrm{DAQ}$ boards collect sufficient data for averaging to track the fastest scintillation effects in the signals. We performed a cubic polynomial fit and calculated the standard deviation for both output signals from SSMF [Fig. 5(a)] and large area detector [Fig. 5(b)] within $15 \mathrm{~s}$ time intervals. Fig. 5(c) shows the cubic polynomial fit and calculated the standard deviation for signal from the MM fiber detector, which is collected via GPIB with a $500 \mathrm{~ms}$ sampling rate. The cubic polynomial fit provides a reasonable average trend for the data. The normalized variances $\sigma_{\text {dust-scintillation }}^{2} / V_{\text {initial }}^{2}$ for different concentrations of the dust 


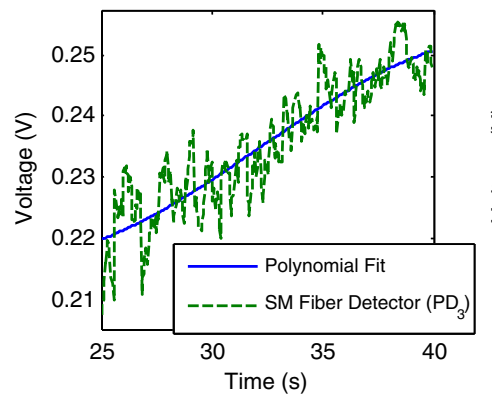

(a)

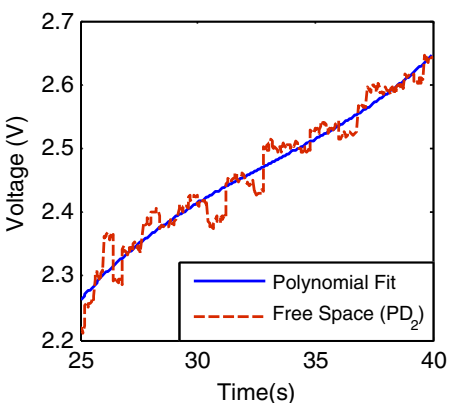

(b)

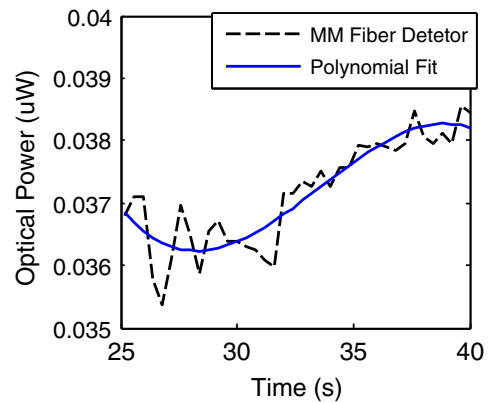

(c)

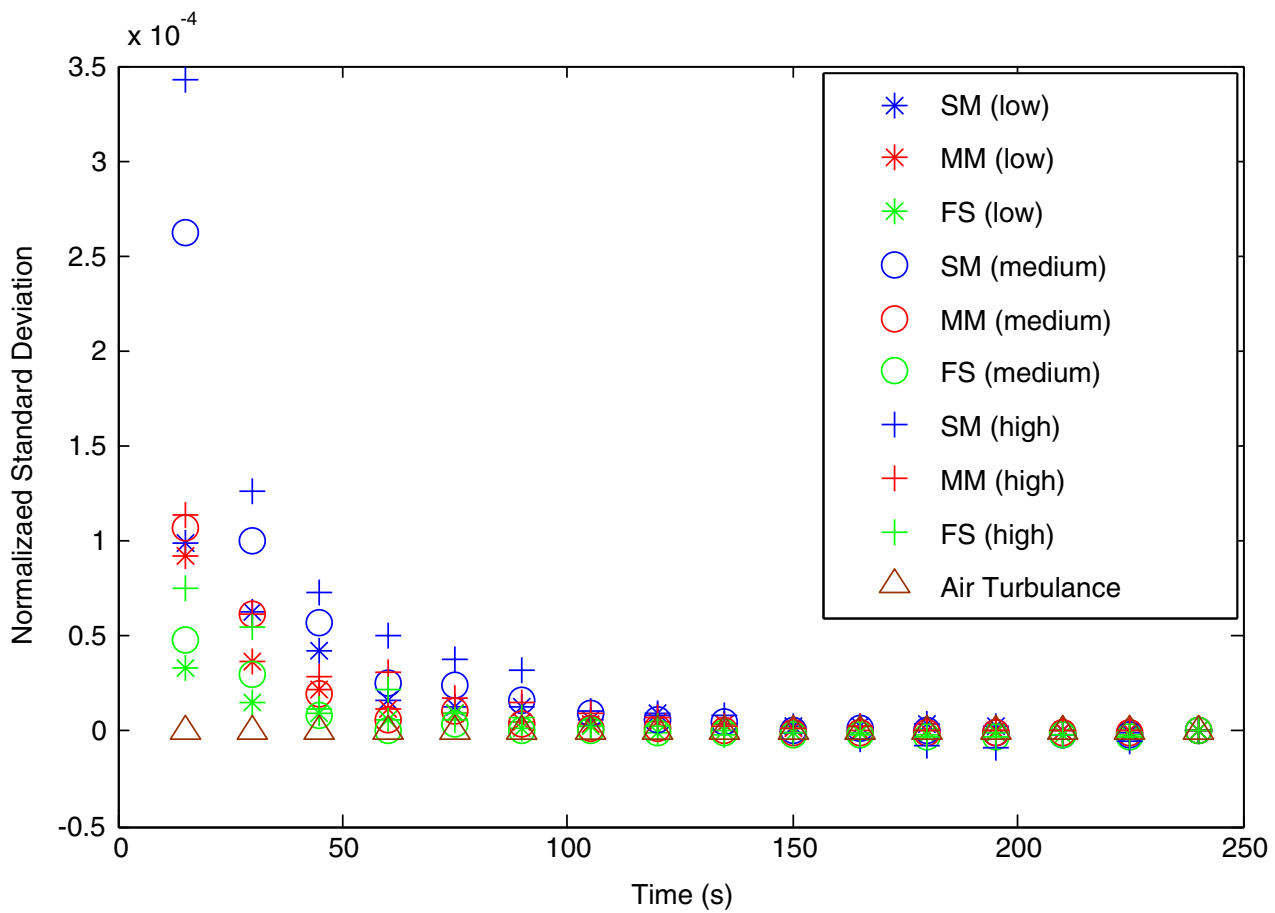

(d)

Fig. 5. (Color online) (a) Output signal of single mode fiber detector with cubic polynomial fit. (b) Output signal of free space detector with cubic polynomial fit. (c) Output signal of MM fiber detector with cubic polynomial fit. (d) Normalized standard deviation of different amount of dust for single mode fiber detector, MM fiber detector, and free space detector.

are calculated according to Eq. (2) and plotted in Fig. 5(d). The brown triangles show as a reference the impact of air turbulence in the chamber on the detector signal when no dust is launched. They are comparably small but indicate that the scintillation effects observable in dust clouds are due to local, time-dependent variations in the dust particle density as opposed to air turbulence. Apparently, the variations in the power coupled into SSMF with small aperture (PD_3) are more pronounced compared to the IR power detected by large area free space detector (PD_2) and MM fiber detector, which indicates that scintillation effects due to the presence of dust particles are observable on the IR link. For the single mode fiber detector, higher dust amounts produce a higher variance. For the free space detector and MM fiber detector, the normalized variance of the output power is smaller than the one of the SSMF detector and it seems to be related to the dust concentration, although our measurement results do not clearly support such a statement. The reason is that scintillation effects are more pronounced for the small aperture detector PD_3 than for the large area detector PD_2 and MM fiber detector.

\section{SIMULATION RESULTS OF THZ ATTENUATION}

In this section, the particle concentration as the function of time is estimated using the evolution of the attenuation in the IR channel according to Beers-Lambert law [19] and Mie scattering theory [20]. Using the inferred time-dependent particle concentration, the $\mathrm{THz}$ attenuation as a function of time is simulated by Mie scattering theory. A comparison of experimental and simulation results shows good agreement.

According to the Beers-Lambert Law, the attenuation of laser radiation through atmosphere can be expressed as

$$
\tau(\lambda, R)=\frac{P(\lambda, R)}{P(\lambda, 0)}=e^{-\gamma(\lambda) R}=e^{-\sigma_{s} N_{s} R},
$$

where $P(\lambda, R)$ is optical power at the distance $R, P(\lambda, 0)$ is the initially emitted optical power, $\gamma(\lambda)$ is the extinction coefficient (per unit of length), $\sigma_{s}$ is the total extinction cross section, and $N_{s}$ is particle concentration. 
The total extinction cross sections can be calculated assuming spherical particles using [1]

$$
\sigma_{s}(v)=\frac{c^{2}}{2 \pi v^{2}} \sum_{m=1}^{\infty}(2 m+1) \operatorname{Re}\left(a_{m}+b_{m}\right),
$$

where $c$ is the speed of light, $v$ is the light frequency, and $a_{m}$, $b_{m}$ are coefficient of the infinite summation such that

$$
\begin{aligned}
a_{m} & =\frac{\psi_{m}^{\prime}(y) \psi_{m}(x)-n \psi_{m}(y) \psi_{m}^{\prime}(x)}{\psi_{m}^{\prime}(y) \zeta_{m}(x)-n \psi_{m}(y) \zeta_{m}^{\prime}(x)} b_{m} \\
& =\frac{n \psi_{m}^{\prime}(y) \psi_{m}(x)-\psi_{m}(y) \psi_{m}^{\prime}(x)}{n \psi_{m}^{\prime}(y) \zeta_{m}(x)-\psi_{m}(y) \zeta_{m}^{\prime}(x)},
\end{aligned}
$$

where

$$
\begin{aligned}
& \psi_{m}(z)=z j_{m}(z) \\
& \zeta_{m}(z)=z h_{m}^{(2)}(z) .
\end{aligned}
$$

Here, $j_{m}(z)$ and $h_{m}^{(2)}(z)$ are spherical Bessel functions of the first kind and third kind, respectively. The size parameter $z$ can be defined as either $x=(2 \pi v r / c)$ or $y=(2 \pi v r / c)$, where $r$ is the radius of the spherical particle and $n$ is refractive index of the particle. The real index of refraction of bentonite is about 1.5 in the IR range and 1.54 at $625 \mathrm{GHz}$.

From Eq. (3), the particle concentration $N_{s}$ can be calculated as

$$
N_{s}=\frac{-\ln \left(\frac{P(\lambda, R)}{P(\lambda, 0)}\right)}{R \sigma_{s}} .
$$

The attenuation is defined as

$$
\text { att_dB }=10 \times \log _{10} \frac{P(\lambda, 0)}{P(\lambda, R)} .
$$

According to the Eqs. (7) and (8), the relationship between $N_{s}$ and the attenuation in $\mathrm{dB}$ can be expressed as

$$
N_{s}=\frac{\text { att } \_\mathrm{dB} \times \ln 10}{10 \times R \times \sigma_{s}} .
$$

For the IR signal with a wavelength around $1.5 \mu \mathrm{m}$ (200 $\mathrm{THz}$ ), the corresponding particle size parameter and the extinction cross section are $z=18.07$ and $\sigma_{s}=1.38 \times 10^{-10} \mathrm{~m}^{2}$, respectively. When using the IR attenuation data of dust particles with concentration at $4 \times 10^{9} / \mathrm{m}^{3}$ [Fig. 3(a)] and substituting an extinction cross section of $\sigma_{s}=1.38 \times 10^{-10} \mathrm{~m}^{2}$ in Eq. (9), the particle concentration as a function of time can be calculated and plotted as shown in Fig. 6(a). The particle concentration varies from $4 \times 10^{9} / \mathrm{m}^{3}$ to almost 0 . Using Eqs. (4), (5), and (6), the THz attenuation as a function of time is calculated. Figure $6(\mathrm{~b})$ shows the comparison of the experimental attenuation of $\mathrm{THz}$ at different times (corresponding to different particle densities of dust), with the corresponding simulation results. The solid line shows the experimental attenuation after applying an 11-point averaging, which reduces the quantization noise of the data. The noise reduction allows one to better visualize the exponential decay in time by eliminating the effect of limited bit resolution of $\mathrm{THz}$ power. The experimental attenuations are in good agreement with the simulation considering the relatively rough quantization of the recorded THz. In summary, the time evolution of the
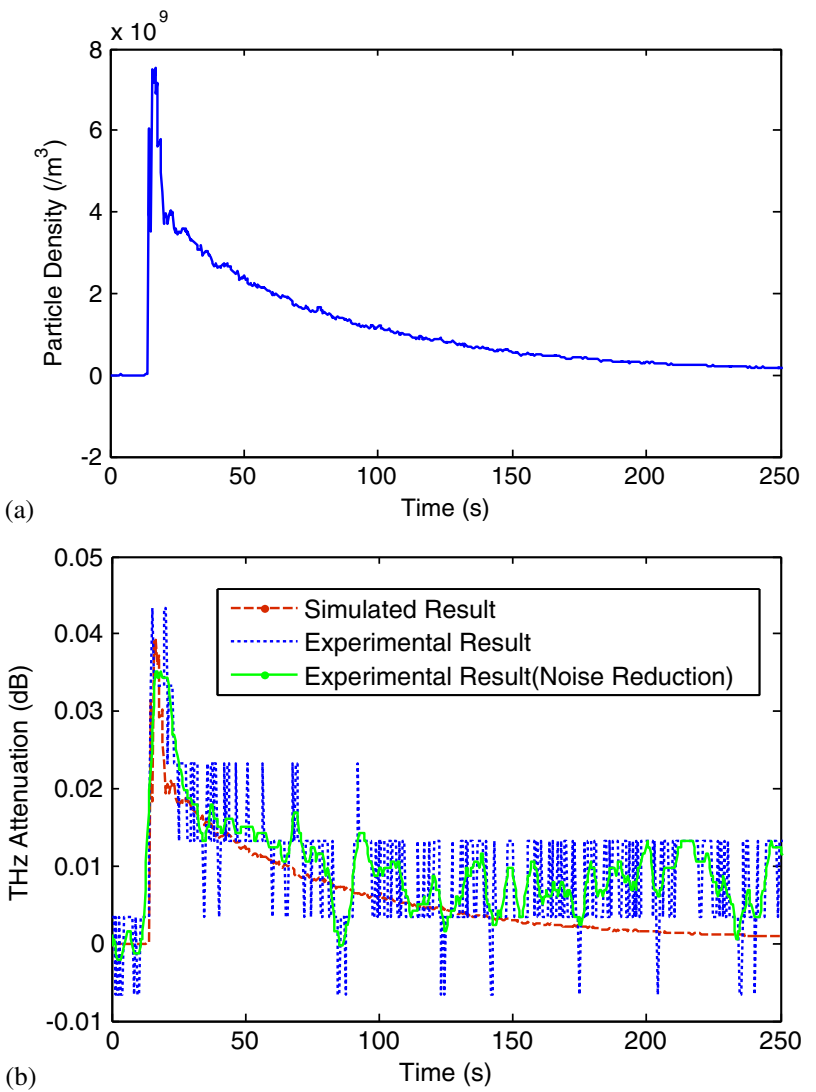

(b)

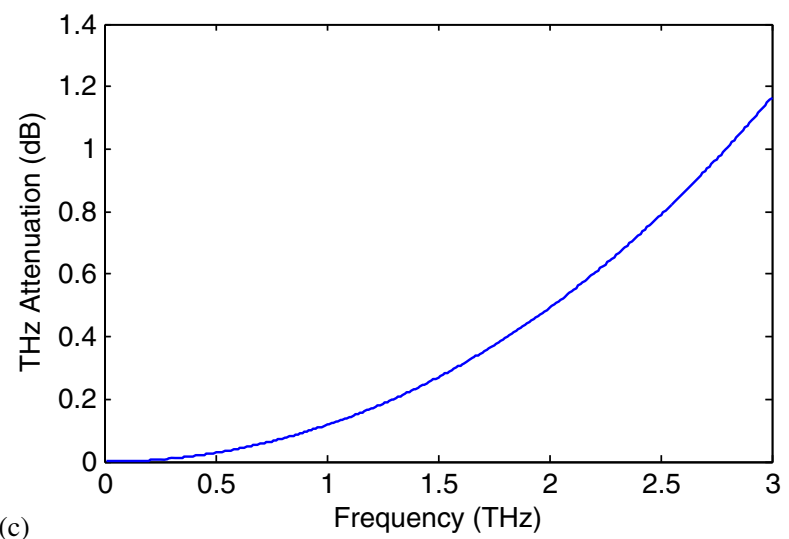

(c)

Fig. 6. (Color online) (a) The particle density with time. (b) Comparison of experimental results, experimental results after undergoing a smoothing algorithm and simulation results of THz attenuation. (c) Predicted THz and sub-THz attenuation duo to bentonite particles (particle density: $4 \times 10^{9} / \mathrm{m}^{3}, 4.3 \mu \mathrm{m}$ particle radius, and a real index of 1.54 ).

$\mathrm{THz}$ attenuation is consistent with the slow time-dependent change in the average particle density of the dust. We also estimate the attenuation due to dust particles from 0.1 to $3 \mathrm{THz}$, which shown in Fig. 6(c).

Since scintillation and speckle effects are an optical path length effect as we discussed in Section 1 , they are expected to be significantly smaller for the $\mathrm{THz}$ beam $(625 \mathrm{GHz})$ than for the IR. The signal fluctuation of the IR output is around $0.2 \mathrm{~dB}$; consequently, the signal fluctuations of $\mathrm{THz}$ are expected to be less than $0.01 \mathrm{~dB}$ from the theoretical estimation, which is beyond our limit of detection. We do not observe the scintillation effects of $\mathrm{THz}$ signals, which is consistent with our theoretical predictions. 


\section{CONCLUSION}

An IR beam at $1.5 \mu \mathrm{m}$ wavelength is superimposed with a $\mathrm{THz}$ beam (625 GHz) carrying the same data load, and propagates through aerosol conditions. Optical power attenuation and BER degradations are measured, analyzed, and compared. Under identical dust concentrations, the IR signal is significantly more attenuated than the $\mathrm{THz}$ signal, which shows almost no degradation from the channel perturbation. Different dust concentrations $\left(2.5 \times 10^{9} / \mathrm{m}^{3}, 4 \times 10^{9} / \mathrm{m}^{3}\right.$, and $6.5 \times 10^{9} / \mathrm{m}^{3}$ ) are simulated in the chamber to generate attenuation for the IR signal in the few $\mathrm{dB}$ range. As the dust loading becomes heavier (and extinction increases), the attenuation becomes more severe in the IR range. However, the impact on the $\mathrm{THz}$ channel is minimal. We also demonstrate that the IR signal is impaired by both attenuation and scintillation effects in the dust cloud. However, the minor degradation of the $\mathrm{THz}$ links due to dust can be explained assuming that the only effect of the dust on the link is to increase the average attenuation in proportion to the dust cloud's particle density, which is in agreement with theoretical simulation. Experimental results presented in this paper indicate that the next step toward the characterization of $\mathrm{THz}$ atmospheric attenuation is to extend our transmission range and outdoor measurement.

\section{ACKNOWLEDGMENTS}

This material is based upon work supported by the National Science Foundation under Grant No. ECCS-1102222.

\section{REFERENCES}

1. J. Federici and L. Moeller, "Review of terahertz and subterahertz wireless communications," J. Appl. Phys. 107, 111101 (2010).

2. T. Kleine-Ostmann and T. Nagatsuma, "A review on terahertz communications research,” J. Infrared Millim. Terahertz Waves 32, 143-171 (2011)

3. S. Cherry, "Edholm's law of bandwidth," IEEE Spectrum 41(7), 58-60 (2004).

4. L. Moeller, J. Federici, A. Sinyukov, C. Xie, H. C. Lim, and R. C. Giles, "Data encoding on terahertz signals for communication and sensing," Opt. Lett. 33, 393-395 (2008).

5. H.-J. Song, K. Ajito, A. Hirata, A. Wakatsuki, Y. Muramoto, T. Furuta, N. Kukutsu, T. Nagatsuma, and Y. Kado, " 8 Gbit/s wireless data transmission at $250 \mathrm{GHz}$," Electron. Lett. 45, 1121-1122 (2009).
6. A. Hirata, T. Kosugi, H. Takahashi, J. Takeuchi, K. Murata, N. Kukutsu, Y. Kado, S. Okabe, T. Ikeda, F. Suginosita, K. Shogen, H. Nishikawa, A. Irino, T. Nakayama, and N. Sudo, "5.8 km 10 Gbps data transmission over a $120 \mathrm{GHz}$-band wireless link," in IEEE International Conference on Wireless Information Technology and Systems (IEEE, 2010), pp. 1-4.

7. L. Moeller, J. Federici, and K. Su, "2.5 Gbit/s duobinary signalling with narrow bandwidth 0.625 terahertz source," Electron. Lett. 47, 856-858 (2011).

8. C. Jansen, R. Piesiewicz, D. Mittleman, T. Kurner, and M. Koch, "The Impact of reflections from stratified building materials on the wave propagation in future indoor terahertz communication systems," IEEE Trans. Antennas Propag. 56, 1413-1419 (2008).

9. Y. Yang, A. Shutler, and D. Grischkowsky, "Measurement of the transmission of the atmosphere from 0.2 to $2 \mathrm{THz}$," Opt. Express 19, 8830-8838 (2011).

10. T. Utsunomiya and M. Sekine, "Rain attenuation at $103 \mathrm{GHz}$ in millimeter wave ranges," Int. J. Infrared Millim. Waves 26, 1651-1660 (2005).

11. A. Hirata, R. Yamaguchi, H. Takahashi, T. Kosugi, K. Murata, N. Kukutsu, and Y. Kado, "Effect of rain attenuation for a $10 \mathrm{~Gb} / \mathrm{s}$ 120 GHz-band millimeter wave wireless link," IEEE Trans. Microwave Theor. Tech. 57, 3099-3105 (2009).

12. S. Ishii, S. Sayama, and T. Kamei, "Measurement of rain attenuation in terahertz wave range," Wireless Eng. Technol. 2, 119-124 (2011).

13. R. Yamaguchi, A. Hirata, T. Kosugi, H. Takahashi, N. Kukutsu, T. Nagatsuma, Y. Kado, H. Ikegawa, H. Nishikawa, and T. Nakayama, "10 Gb/s MMIC wireless link exceeding 800 meters," in Radio and Wireless Symposium (IEEE, 2008), pp. 695-698.

14. K. Su, L. Moeller, R. B. Barat, and J. F. Federici, "Experimental comparison of performance degradation from terahertz and infrared wireless links in fog," J. Opt. Soc. Am. A 29, 179-184 (2012).

15. C. M. Mann, "Towards terahertz communications systems," in Terahertz Sources and Systems, R. Miles, P. Harrison, and D. Lippens, eds. (Academic, 2001).

16. A. Lender, "The duobinary technique for high-speed data transmission," IEEE Trans. Commun. Electron. 82, 214-218 (1963).

17. D. Penninckx, M. Chbat, L. Pierre, and J. P. Thiery, "The phaseshaped binary transmission (PSBT): a new technique to transmit far beyond the chromatic dispersion limit," IEEE Photon. Technol. Lett. 9, 259-261 (1997).

18. T. Mizuochi, "Recent progress in forward error correction and its interplay with transmission impairments," IEEE J. Sel. Top. Quantum Electron. 12, 544-554 (2006).

19. H. Weichel, Laser Beam Propagation in the Atmosphere (Academic, 1990).

20. C. F. Bohren and D. R. Huffman, Absorption and Scattering of Light by Small Particles (Academic, 1983). 